\title{
Tilings and Submonoids of Metabelian Groups
}

\author{
Markus Lohrey $^{1}$ and Benjamin Steinberg ${ }^{2, \star}$ \\ 1 Universität Leipzig, Institut für Informatik, Germany \\ 2 School of Mathematics and Statistics, Carleton University, ON, Canada \\ lohrey@informatik.uni-leipzig.de, bsteinbg@math.carleton.ca
}

\begin{abstract}
In this paper we show that membership in finitely generated submonoids is undecidable for the free metabelian group of rank 2 and for the wreath product $\mathbb{Z} \succ(\mathbb{Z} \times \mathbb{Z})$. We also show that subsemimodule membership is undecidable for finite rank free $(\mathbb{Z} \times \mathbb{Z})$-modules. The proof involves an encoding of Turing machines via tilings. We also show that rational subset membership is undecidable for two-dimensional lamplighter groups.
\end{abstract}

\section{Introduction}

Two of the classical group theoretic decision problems are the word problem and the generalized word problem. Suppose $G$ is a finitely generated group with finite generating set $\Sigma$ and put $\Sigma^{ \pm}=\Sigma \cup \Sigma^{-1}$. Let $\pi:\left(\Sigma^{ \pm}\right)^{*} \rightarrow G$ be the canonical projection from the free monoid on $\Sigma^{ \pm}$onto $G$. The word problem asks to determine algorithmically given an input word $w \in\left(\Sigma^{ \pm}\right)^{*}$, whether $\pi(w)=1$. An algorithm for the (uniform) generalized word problem takes as input finitely many words $w, w_{1}, \ldots, w_{n} \in\left(\Sigma^{ \pm}\right)^{*}$ and answers whether $\pi(w) \in\left\langle\pi\left(w_{1}\right), \ldots, \pi\left(w_{n}\right)\right\rangle$. Two more general problems that have received some attention in recent years are the submonoid membership $[13,15]$ and the rational subset membership problems [11-13,17, 19].

The (uniform) submonoid membership problem for $G$ takes as input a finite list of words $w, w_{1}, \ldots, w_{n} \in\left(\Sigma^{ \pm}\right)^{*}$ and asks the question is $\pi(w) \in\left\{\pi\left(w_{1}\right), \ldots, \pi\left(w_{n}\right)\right\}^{*}$ (where if $X \subseteq G$, then $X^{*}$ denotes the submonoid generated by $G$ ). For example, $g \in G$ has finite order if and only if $g^{-1} \in g^{*}$ and so decidability of membership in cyclic submonoids allows one to compute the order of an element. Of course, decidability of submonoid membership implies decidability of the generalized word problem. In [13] the authors provided the first example of a group with decidable generalized word problem and undecidable submonoid membership problem, namely the right-angled Artin group (or graph group) whose associated graph is a path of length 3 .

The rational subset membership problem for $G$ is the following algorithmic problem: given as input a word $w \in\left(\Sigma^{ \pm}\right)^{*}$ and a finite automaton $\mathscr{A}$ over $\left(\Sigma^{ \pm}\right)^{*}$, determine whether $\pi(w) \in \pi(L(\mathscr{A}))$. Of course, this is the most general of the problems we have been discussing, and is therefore the easiest to prove undecidable. A subset of the group $G$ is called rational if it is of the form $\pi(L(\mathscr{A}))$ for some finite automaton $\mathscr{A}$. This definition does not depend on the generating set. The study of rational subsets of groups

\footnotetext{
* The authors would like to acknowledge the support of DFG Mercator program. The second author is also supported by an NSERC grant.
} 
goes back a long way, beginning with free groups [5] and commutative groups [10]. Other early references include $[2,7]$. The largest known class of groups with decidable rational subset membership problem can be found in [13], where one also finds a complete classificaiton of graph groups with decidable rational subset membership problem.

It is easy to see that if $L_{1}, L_{2}$ are rational subsets of $G$, then $L_{1} \cap L_{2} \neq \emptyset$ if and only if $1 \in L_{1} L_{2}^{-1}$ and the latter product is a rational subset of $G$. There are many monoids embeddable in groups with undecidable rational subset intersection emptiness problems; one such example was exploited in [13]. An easier example is the following. Let $M$ be a free monoid on two-generators. Then the undecidability of the Post correspondence problem implies that there is a fixed finitely generated submonoid $N$ of $M \times M$ so that it is undecidable given an element $(u, v) \in M \times M$ whether $(u, v) N \cap \Delta \neq \emptyset$ where $\Delta$ is the diagonal submonoid of $M \times M$. Therefore, if $G$ is a finitely generated group containing $M \times M$, then $(u, v) N \cap \Delta \neq \emptyset$ if and only if $(u, v) \in \Delta N^{-1}$ and so rational subset membership is undecidable for a fixed rational subset of such a group. For instance, Thompson's group $F$ contains a direct product of two free monoids of rank 2 and so has undecidable rational subset membership problem. It was shown by [19] that, for any nilpotency class $c \geq 2$, there is a rank $r$ so that the free nilpotent group of class $c$ and rank $r$ has undecidable rational subset membership problem via an encoding of Hilbert's tenth problem. On the other hand, from the subgroup separability of polycyclic groups [14] it follows that the latter have a decidable generalized word problem. A more practical algorithm can be found in [3].

It is well known that free solvable groups of rank 2 and derived length at least 3 have undecidable generalized word problem [24]. On the other hand, all finitely generated metabelian groups have a decidable generalized word problem [21,22]. It is therefore natural to consider metabelian groups for the submonoid and rational subset membership problems. In this paper we show that there is a fixed finitely generated submonoid of the free metabelian group of rank 2 with undecidable membership problem. The same result is also established for the wreath product $\mathbb{Z} \imath(\mathbb{Z} \times \mathbb{Z})$. The proof is via a reduction to the membership problem for finitely generated subsemimodules of free $(\mathbb{Z} \times \mathbb{Z})$-modules of finite rank. This latter problem we prove undecidable by interpreting it as a particular tiling problem that we show to be undecidable via a direct encoding of a Turing machine.

The paper ends by showing that membership in rational subsets of the metabelian group $\mathbb{Z} / n \mathbb{Z} \imath(\mathbb{Z} \times \mathbb{Z})$ is undecidable using essentially the same tiling problem. It is left open whether this group has a decidable submonoid membership problem. At the moment, there are no examples of groups for which the submonoid membership problem is decidable, but the rational subset membership problem is undecidable. Some further algorithmic results concerning metabelian groups can be found in $[4,16,20]$.

\section{The subsemimodule membership problem}

Fix a group $G$. Recall that a (left) $G$-module is an abelian group $M$ equipped with a left action of $G$ by automorphisms. Equivalently, a $G$-module is a module for the group ring $\mathbb{Z} G$. One can extend this definition to obtain the notion of a $G$-semimodule. By a 
$G$-semimodule, we mean a commutative monoid $N$ equipped with a left action of $G$ by automorphisms. Equivalently, we are speaking of semimodules for the group semiring $\mathbb{N} G$. If $X$ is a subset of a $G$-module, then $\mathbb{Z} G \cdot X$ will denote the submodule generated by $X$ and $\mathbb{N} G \cdot X$ will denote the subsemimodule generated by $X$.

Let us now formulate the membership problem for semimodules. Informally, the problem is given a fixed finitely generated $G$-module $M$, can one determine algorithmically membership in finitely generated subsemimodules of $M$. Of course, to make this a well-defined algorithmic problem we need to describe how to represent elements of the module.

Assume now that our group $G$ is generated by a finite set $\Sigma$ and denote by $\Sigma^{ \pm}$ the set $\Sigma \cup \Sigma^{-1}$. Let $\mathbb{Z} \Sigma^{ \pm}$be the ring of integral polynomials in non-commuting variables $\Sigma^{ \pm}$(that is the free ring on $\Sigma^{ \pm}$). There is as usual a canonical surjection $\pi: \mathbb{Z} \Sigma^{ \pm} \rightarrow \mathbb{Z} G$ induced by evaluating words in $G$.

Let $M$ be a finitely generated $G$-module with generating set $B$. We can view it as a $\mathbb{Z} \Sigma^{ \pm}$-module via $\pi$. Let $\widetilde{M}$ be the free $\mathbb{Z} \Sigma^{ \pm}$-module on $B$. Then there is a canonical projection $\rho: \widetilde{M} \rightarrow M$ sending $B$ to $B$. The idea then is that we can represent elements of $M$ by elements of $\widetilde{M}$. The (uniform) subsemimodule membership problem then takes as input a finite subset $F$ of $\widetilde{M}$ and an element $x \in \widetilde{M}$. The output is whether $\rho(x) \in$ $\mathbb{N} G \cdot \rho(F)$. It should be noted that for $G=1$ the subsemimodule membership problem corresponds to integer programming, which is a classical NP-complete problem.

Our interest in the subsemimodule membership problem stems from an easy encoding of it into the submonoid membership problem for semidirect products.

Lemma 1. Let $G$ be a group with generating set $\Sigma$ and let $M$ be a $G$-semimodule generated by a subset $B$. Then the semidirect product $M \rtimes G$ is generated as a monoid by $\Sigma^{ \pm} \cup B$ via the map $a \mapsto(0, a)$ for $a \in \Sigma^{ \pm}$and $b \mapsto(b, 1)$ for $b \in B$. In particular, if $G$ and $M$ are finitely generated, then so is $M \rtimes G$.

Proof. As a monoid $M$ is generated by all elements of the form $g b$ with $g \in G, b \in B$. But $(0, g)(b, 1)\left(0, g^{-1}\right)=(g b, 1)$. It follows that $\Sigma^{ \pm} \cup B$ is a monoid generating set for $M \rtimes G$.

In light of Lemma 1, we immediately obtain the following result.

Proposition 2. Let $G$ be a finitely generated group and $M$ a finitely generated $G$ module with an undecidable subsemimodule membership problem (for a fixed subsemimodule $N$ ). Then $M \rtimes G$ has an undecidable submonoid membership problem (for the fixed submonoid $N \rtimes G$ ).

Proof. The membership of $(m, 1)$ in $N \rtimes G$ is evidently equivalent to the membership of $m \in N$. Let us just mention how one effectively transforms input from the subsemimodule problem to the submonoid membership problem. Suppose $\Sigma$ is a generating set for $G$ and $B$ is a generating set for $M$. Let $\widetilde{M}$ and $\rho$ be as before Lemma 1 . Then, for $w \in\left(\Sigma^{ \pm}\right)^{*}, b \in B$ and $n \in \mathbb{Z}$, the element $(\rho(n w b), 1)$ is represented in the (group) generating set $\Sigma \cup B$ for $M \rtimes G$ by the word $\left(w b w^{-1}\right)^{n}$. In this way, we can encode representatives of the module as words in $\left((\Sigma \cup B)^{ \pm}\right)^{*}$. 
If $G$ is a group, the semidirect product $\mathbb{Z} G \rtimes G$ is the same thing as the (restricted) wreath product $\mathbb{Z} \imath G$. Now if $H$ is a subgroup of $G$ of index $m$, then it is well known that $\mathbb{Z} G$ is a free $\mathbb{Z} H$-module of rank $m$ [9]. More precisely, if $T=\left\{g_{1}, \ldots, g_{m}\right\}$ is a complete set of right coset representatives of $H$ in $G$, then $T$ is a basis for $\mathbb{Z} G$ as a free left $\mathbb{Z} H$ module. Consequently, we have the following lemma.

Lemma 3. Suppose that $H$ is a subgroup of $G$ of index $m$ and $M$ is a free $\mathbb{Z} H$-module of rank at most $m$. Then $M \rtimes H$ embeds as a subgroup of $\mathbb{Z} \imath G$.

Proof. Clearly $\mathbb{Z} G \rtimes H \leq \mathbb{Z} G \rtimes G=\mathbb{Z} \imath G$. Since $\mathbb{Z} G$ is a free $\mathbb{Z} H$-module of rank $m$, it follows $M \leq \mathbb{Z} G$ and so we are done.

The main technical result of this paper is the following theorem.

Theorem 4. There is a free $(\mathbb{Z} \times \mathbb{Z})$-module of finite rank with an undecidable subsemimodule membership problem for a fixed finitely generated subsemimodule.

As a corollary, we obtain that $\mathbb{Z} \imath(\mathbb{Z} \times \mathbb{Z})$ has an undecidable submonoid membership problem. This should be contrasted with the generalized word problem, which is solvable in any finitely generated metabelian group [21,22]. It should be noted that the submodule membership problem is decidable for free $(\mathbb{Z} \times \mathbb{Z})$-modules [23], and this is what underlies the positive solution to the generalized word problem for metabelian groups in [21,22].

Corollary 5. The submonoid membership problem is undecidable for $\mathbb{Z} \imath(\mathbb{Z} \times \mathbb{Z})$ for a fixed finitely generated submonoid.

Proof. By Theorem 4, there is a free $(\mathbb{Z} \times \mathbb{Z})$-module $M$ of some rank $m$ with undecidable subsemimodule membership problem for a fixed subsemimodule. Proposition 2 then implies that $M \rtimes(\mathbb{Z} \times \mathbb{Z})$ has undecidable submonoid membership for a fixed finitely generated submonoid. Now $\mathbb{Z} \times \mathbb{Z}$ has a subgroup of index $m$ isomorphic to it, e.g., $m \mathbb{Z} \times \mathbb{Z}$. Lemma 3 then implies $M \rtimes(\mathbb{Z} \times \mathbb{Z})$ embeds in $\mathbb{Z} \imath(\mathbb{Z} \times \mathbb{Z})$, completing the proof.

Recall that a group $G$ is metabelian if it is solvable of derived length 2, or equivalently if commutators in $G$ commute. Our next goal is to show that the free metabelian group of rank 2 has an undecidable submonoid membership problem for a fixed submonoid. Since it is known that free non-cyclic solvable groups of derived length 3 or higher have undecidable generalized word problem [24], this will show that the submonoid membership problem is undecidable for free non-abelian solvable groups of any derived length.

We need to recall a description of the free metabelian group of rank 2, which is a special case of a more general result of Almeida [1]; see also [16]. In what follows we will work with the Cayley-graph $\Gamma$ of the group $\mathbb{Z} \times \mathbb{Z}$. More precisely, the set of vertices of $\Gamma$ is $\mathbb{Z} \times \mathbb{Z}$ and the set of (undirected) edges is

$$
\mathscr{E}=\{\{(p, q),(r, s)\}|p, q, r, s \in \mathbb{Z},| u-x|+| v-y \mid=1\} .
$$


For $e=\{(p, q),(r, s)\} \in \mathscr{E}$ and $(a, b) \in \mathbb{Z} \times \mathbb{Z}$, we define the translation $e+(a, b)=$ $\{(p+a, q+b),(r+a, s+b)\} \in \mathscr{E}$. Let $\Sigma=\{x, y\}$ and label edges in $\Gamma$ of the form $\{(p, q),(p+1, q)\}$ (resp. $\{(p, q),(p, q+1)\}$ ) with $x$ (resp. $y$ ); the reverse edges are labeled with $x^{-1}$ (resp. $y^{-1}$ ). Let $M_{2}$ be the free metabelian group generated by $\Sigma$. Then two words $u, v$ in $\left(\Sigma^{ \pm}\right)^{*}$ represent the same element of $M_{2}$ if and only if they map to the same element of the free abelian group of rank 2 and the paths traversed by $u$ and $v$ in the Cayley graph $\Gamma$ of $\mathbb{Z} \times \mathbb{Z}$ use each edge the same number of times (where backwards traversals are counted negatively). Equivalently, a word $w$ represents the identity in $M_{2}$ if and only if it labels a closed path in $\Gamma$ at the origin that maps to the trivial element of the homology group $H_{1}(\Gamma)$. A word $w$ represents an element of the commutator subgroup $\left[M_{2}, M_{2}\right]$ if and only if it reads a closed loop in $\Gamma$ at the origin. Thus $\left[M_{2}, M_{2}\right]$ can be identified with $H_{1}(\Gamma)$ as a $(\mathbb{Z} \times \mathbb{Z})$-module by mapping a word $w$ reading a loop at the origin to the element of $H_{1}(\Gamma)$ represented by that loop. As a $(\mathbb{Z} \times \mathbb{Z})$-module, it is free of rank 1 generated by the commutator $[x, y]=x y x^{-1} y^{-1}$, which corresponds to

$$
c=\{(0,0),(1,0)\}+\{(1,0),(1,1)\}-\{(1,1),(0,1)\}-\{(0,1),(0,0)\}
$$

under our identification of $\left[M_{2}, M_{2}\right]$ with $H_{1}(\Gamma)$. The easiest way to see that $c$ is a free generator is to view $\Gamma$ as the 1-skeleton of $\mathbb{R}^{2}$ with the cell complex structure whose 2 -cells are the squares of side length 1 bounded by $\Gamma$. The fact that $H_{2}\left(\mathbb{R}^{2}\right)=$ $0=H_{1}\left(\mathbb{R}^{2}\right)$ says exactly that the boundary map from the free abelian group on the cells to $H_{1}(\Gamma)$ (which can be identified with $Z_{1}\left(\mathbb{R}^{2}\right)$ ) is an isomorphism. Moreover, the boundary map is actually a homomorphism of $(\mathbb{Z} \times \mathbb{Z})$-modules since the action of $\mathbb{Z} \times \mathbb{Z}$ on $\mathbb{R}^{2}$ is by cellular maps. Since $\mathbb{Z} \times \mathbb{Z}$ acts freely and transitively on the cells, it follows that $H_{1}(\Gamma)$ is freely generated by $c$.

Fix now $m>0$ and consider $H=\left\langle x^{m}, y\right\rangle \leq M_{2}$. First note that the image of $H$ in $M_{2} /\left[M_{2}, M_{2}\right]=\mathbb{Z} \times \mathbb{Z}$ is the subgroup $m \mathbb{Z} \times \mathbb{Z}$, which must therefore be the abelianization of $H$ as it is free of rank 2 and $H$ is 2-generated. Thus $[H, H]=$ $\left[M_{2}, M_{2}\right] \cap H$. Moreover, $[H, H]$ is the $m \mathbb{Z} \times \mathbb{Z}$-submodule of $\left[M_{2}, M_{2}\right]$ generated by

$$
c^{\prime}=\sum_{i=0}^{m-1}(c+(i, 0)) .
$$

Indeed, the elements of $[H, H]$ are the homology classes in $H_{1}(\Gamma)$ of closed loops in the grid with vertex set $m \mathbb{Z} \times \mathbb{Z}$. If we make $\mathbb{R}^{2}$ into a cell complex by using the squares bounded by this grid, then the same argument as above shows that $[H, H]$ is freely generated as an $(m \mathbb{Z} \times \mathbb{Z})$-module by the boundary of the square with vertices

$$
(0,0),(m, 0),(0,1),(m, 1) .
$$

But this is exactly $c^{\prime}$.

Now as an $(m \mathbb{Z} \times \mathbb{Z})$-module, $\left[M_{2}, M_{2}\right]$ is free on $\{c+(i, 0) \mid 0 \leq i \leq m-1\}$. But we can then change the basis to the set

$$
\{c+(i, 0) \mid 0 \leq i \leq m-2\} \cup\left\{c^{\prime}\right\} .
$$




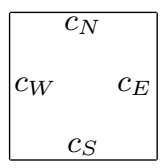

Fig. 1. The tile $t=\left(c_{N}, c_{E}, c_{S}, c_{W}\right)$

Thus as an $(m \mathbb{Z} \times \mathbb{Z})$-module $\left[M_{2}, M_{2}\right]=F \oplus[H, H]$ where $F$ is free of rank $m-1$. We can exploit this to reduce the subsemimodule membership problem to the submonoid membership problem for $M_{2}$.

Theorem 6. There is a fixed finitely generated submonoid of the free metabelian group of rank 2 with undecidable membership problem.

Proof. By Theorem 4 we can find a free $(\mathbb{Z} \times \mathbb{Z})$-module $M$ of rank $r$ containing a fixed finitely generated subsemimodule $N$ with an undecidable membership problem. Choose $m=r+1$ and set $H=\left\langle x^{m}, y\right\rangle$. We saw above that as an $(m \mathbb{Z} \times \mathbb{Z})$-module we can write $\left[M_{2}, M_{2}\right]=F \oplus[H, H]$ where $F$ is a free $(m \mathbb{Z} \times \mathbb{Z})$-module of rank $r$. Since $m \mathbb{Z} \times \mathbb{Z} \cong \mathbb{Z} \times \mathbb{Z}$, we can of course find a fixed subsemimodule, which we abusively denote $N$, inside of $F$ with an undecidable subsemimodule membership problem. Consider the submonoid $S$ of $M_{2}$ generated by $N$ and $H$. If $B$ is a finite generating set for $N$, then $S$ is generated by $B \cup\left\{x^{m}, x^{-m}, y, y^{-1}\right\}$ since each translate of an element of $B$ by an element of $m \mathbb{Z} \times \mathbb{Z}$ can be obtained via a conjugation by an element of $H$. We claim that $S \cap\left[M_{2}, M_{2}\right]=N \oplus[H, H]$. Notice that $N \cap[H, H]=0$ since $N \leq F$ and $F \cap[H, H]=0$, so $N+[H, H]=N \oplus[H, H]$. The inclusion from right to left is trivial. For the other inclusion, consider a product $g=h_{0} n_{0} \cdots h_{k} n_{k}$ with the $h_{i} \in H$ and the $n_{i} \in N$ belonging to $\left[M_{2}, M_{2}\right]$. Then

$$
\begin{aligned}
g & =\left(h_{0} n_{0} h_{0}^{-1}\right)\left(h_{0} h_{1} n_{1}\left(h_{0} h_{1}\right)^{-1}\right) \cdots\left(h_{0} \cdots h_{k} n_{k}\left(h_{0} \cdots h_{k}\right)^{-1}\right) h_{0} \cdots h_{k} \\
& =n h_{0} \cdots h_{k}
\end{aligned}
$$

with $n \in N$. It follows that $h_{0} \cdots h_{k} \in\left[M_{2}, M_{2}\right] \cap H=[H, H]$ and so we obtain $g \in N \oplus[H, H]$, as required.

So suppose $x \in F$ and we want to decide whether $x \in N$. Then since we have $S \cap\left[M_{2}, M_{2}\right]=N \oplus[H, H] \leq F \oplus[H, H]$, it follows that $x \in N$ if and only if $x \in S$. This completes the proof.

\section{Tiling problems}

There is a classical connection between Turing machines and tiling problems [6, 18]. Here we consider a variant that is most easily translated into the subsemimodule membership problem.

Let $C$ be a finite set of colors with a distinguished color $c_{0} \in C$. A tiling system over $C$ is a set $T \subseteq C^{4}$; its elements are called tiles. We view a tile $t=\left(c_{N}, c_{E}, c_{S}, c_{W}\right)$ as an edge colored square, as shown in Figure 1. We will associate with the tile $t=$ 
$\left(c_{N}, c_{E}, c_{S}, c_{W}\right)$ the following mapping $\llbracket t \rrbracket: \mathscr{E} \times C \rightarrow \mathbb{Z}$ (where $\mathscr{E}$ continues to denote the edge set of the Cayley graph of $\mathbb{Z} \times \mathbb{Z}$ ):

$$
\begin{gathered}
\llbracket t \rrbracket\left(\{(0,0),(1,0)\}, c_{S}\right)=-1 \cdot\left(1-\delta_{c_{S}, c_{0}}\right) \\
\llbracket t \rrbracket\left(\{(1,0),(1,1)\}, c_{E}\right)=1 \cdot\left(1-\delta_{c_{E}, c_{0}}\right) \\
\llbracket t \rrbracket\left(\{(1,1),(0,1)\}, c_{N}\right)=1 \cdot\left(1-\delta_{c_{N}, c_{0}}\right) \\
\llbracket t \rrbracket\left(\{(0,1),(0,0)\}, c_{W}\right)=-1 \cdot\left(1-\delta_{c_{W}, c_{0}}\right) \\
\llbracket t \rrbracket(e, c)=0 \text { in all other cases }
\end{gathered}
$$

where as usual $\delta_{x, y}=1$ when $x=y$ and $\delta_{x, y}=0$ if $x \neq y$. Thus, we color, for instance, the north edge $\{(0,1),(1,1)\}$ of the cell $\{(x, y) \mid 0 \leq x, y \leq 1\}$ with the color $c_{N}$, in case $c_{N} \neq c_{0}$. The sign of the value $\llbracket t \rrbracket(e, c)$ indicates that the north and east (south and west) edge receive a positive (negative) orientation. This will be used below, where we add translates of the maps $\llbracket t \rrbracket$. Edges that are colored by $c_{0}$ receive the value 0 .

Let $f: \mathscr{E} \times C \rightarrow \mathbb{Z}$. We say that $f$ has finite support if the set $f^{-1}(\mathbb{Z} \backslash\{0\})$ is finite. For $(a, b) \in \mathbb{Z} \times \mathbb{Z}$ we define the translate $\tau_{a, b} f: \mathscr{E} \times C \rightarrow \mathbb{Z}$ as the mapping with

$$
\tau_{a, b} f(e, c)=f(e-(a, b), c) \text { for all } e \in \mathscr{E} \text { and } c \in C \text {. }
$$

For two mappings $f_{1}, f_{2}: \mathscr{E} \times C \rightarrow \mathbb{Z}$ we define the sum $f_{1}+f_{2}: \mathscr{E} \times \Gamma \rightarrow \mathbb{Z}$ by $\left(f_{1}+f_{2}\right)(e, c)=f_{1}(e, c)+f_{2}(e, c)$ for all $e \in \mathscr{E}$ and $c \in C$. We denote by 0 the constant mapping taking the value 0 everywhere on $\mathscr{E} \times \Gamma$. The set of all mappings from $\mathscr{E} \times C$ to $\mathbb{Z}$ forms an abelian group under addition. The set of all mappings with finite support is a subgroup of this group.

A tiling sum over $T$ is a sum of the form

$$
f=\sum_{i=1}^{n} \tau_{x_{i}, y_{i}} \llbracket t_{i} \rrbracket,
$$

where $x_{i}, y_{i} \in \mathbb{Z}$ and $t_{i} \in T$ for all $1 \leq i \leq n$. The evaluation of such a sum yields a mapping $f: \mathscr{E} \times C \rightarrow \mathbb{Z}$. Note that one may have $\left(x_{i}, y_{i}\right)=\left(x_{j}, y_{j}\right)$ for $i \neq j$. Intuitively one can think of a tiling sum as putting tiles on certain positions of the grid (one may put several tiles on the same position or even put the same tile several times on the same position). When evaluating the tiling sum, we cancel matching colors on edges, which happens if, e.g., the color on the north side of a tile matches the color on the south side of the tile immediately above it. The distinguished color $c_{0}$ is not involved in this cancellation process. Let us agree to say that the tile $t_{i}$ is placed in position $\left(x_{i}, y_{i}\right)$ in the tiling sum (1). Of course, the same tile may be placed in multiple positions or even multiple times in the same position.

The zero tiling sum problem for a given tiling system $T$ over $C$ is the following computational problem:

INPUT: A mapping $f_{0}: \mathscr{E} \times \Gamma \rightarrow \mathbb{Z}$ with finite support.

QUESTION: Is there a tiling sum $f$ with $f_{0}+f=0$ ? 
Theorem 7. The zero tiling sum problem is undecidable.

Proof. We start with a fixed deterministic Turing machine $M=\left(Q, \Gamma, \Sigma, \delta, q_{0}, q_{f}\right)$ with an undecidable acceptance problem. Here, $Q$ is the set of states, $\Gamma$ is the tape alphabet, $\Sigma \subseteq \Gamma$ is the input alphabet, $q_{0} \in Q$ is the initial state, $q_{f} \in Q$ is the unique accepting state, and $\delta: Q \times \Gamma \rightarrow Q \times \Gamma \times\{L, R\}$ is the transition mapping ( $L$ (resp. $R$ ) means that the head moves left (resp. right)). The blank symbol is $\square \in \Gamma \backslash \Sigma$. We can make the following assumptions on the machine $M$ :

- The tape of $M$ is bounded to the left; that is, the machine never moves to the left of the first cell.

- $M$ terminates if and only if it reaches the accepting state $q_{f}$. In particular, an input $w$ is accepted if and only if $M$ terminates on $w$.

- If $M$ reaches state $q_{f}$ then the whole tape is blank and the head of the machine is scanning the left most cell.

We take the following fixed set of colors:

$$
C=Q \cup \Gamma \cup(Q \times \Gamma) \cup\left\{\rightarrow, \uparrow, \leftarrow, \downarrow,\left\llcorner, \triangleleft, \triangleright, c_{0}\right\} .\right.
$$

Here, $c_{0}$ is the distinguished color. In the following pictures the color $c_{0}$ will be indicated in a tile by a dotted side. Also the pair $(q, a) \in Q \times \Gamma$ will be written $q a$.

The set of tiles $T$ consists of the following tiles, which are inspired by the tiles of the tiling system from [8, Appendix A]:

- Alphabet tiles (for all $a \in \Gamma$ ):

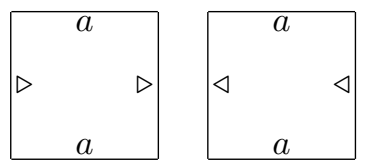

- Merging tiles (for all $a \in \Gamma$ and all $p \in Q$ ):

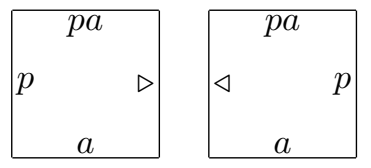

- Action tiles for moves of the machine $M$ :

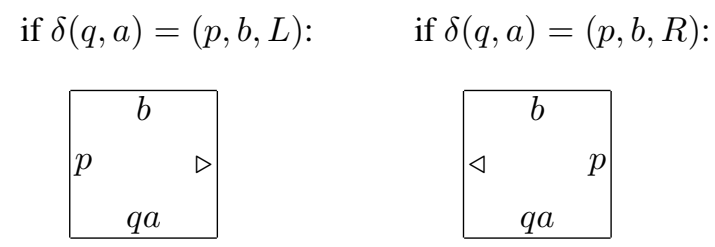


- Boundary tiles (the labels $b_{i}$ are just names that we give to these tiles):

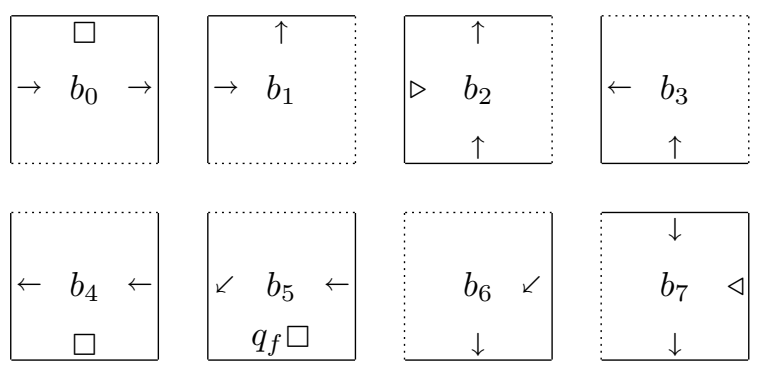

Now, let $w=w_{1} w_{2} \cdots w_{n}$ be an input for the machine $M$ with the $w_{i} \in \Sigma$. We associate with $w$ the following mapping $f_{w}: \mathscr{E} \times C \rightarrow \mathbb{Z}$ :

$$
\begin{aligned}
f_{w}(\{(0,1),(1,1)\}, \downarrow) & =1 \\
f_{w}\left(\{(1,1),(2,1)\}, q_{0} w_{1}\right) & =1 \\
f_{w}\left(\{(i, 1),(i+1,1)\}, w_{i}\right) & =1 \text { for } 2 \leq i \leq n \\
f_{w}(\{(n+1,1),(n+1,0)\}, \rightarrow) & =1 .
\end{aligned}
$$

All other values of $f_{w}$ are 0 , hence $f_{w}$ has finite support. As a diagram, the mapping $f_{w}$ looks as follows:

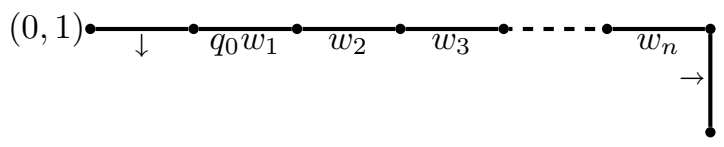

We will show that $M$ accepts the input $w$ if and only if there is a tiling sum $f$ with $f_{w}+f=0$.

First assume that there is such a tiling sum $f$ and let

$$
f=\sum_{i=1}^{N} \tau_{x_{i}, y_{i}} \llbracket t_{i} \rrbracket .
$$

Claim 1. For all $1 \leq i \leq N$, we have both $x_{i}, y_{i} \geq 0$ and either $y_{i} \geq 1$, or $x_{i} \geq n+1$ in (2), i.e., all tiles are placed into the shaded area in Figure 2.

Let $\preceq$ be the componentwise order on $\mathbb{Z} \times \mathbb{Z}$, i.e., $\left(x^{\prime}, y^{\prime}\right) \preceq(x, y)$ if and only if $x^{\prime} \leq x$ and $y^{\prime} \leq y$. In order to deduce a contradiction, assume that there exists a tile of $f$ placed outside the shaded area and suppose that $i$ is chosen so that $\left(x_{i}, y_{i}\right)$ is $\preceq$-minimal with $\tau_{\left(x_{i}, y_{i}\right)} \llbracket t_{i} \rrbracket$ outside of the shaded area. Note that for every tile in $T$, the south or the west edge is colored differently from $c_{0}$. Hence, the south or the west color of tile $t_{i}$ is different from $c_{0}$. In order to match this up, there must exist a tile placed to the south or to the west of $t_{i}$, that is, there must be $1 \leq j \leq N$ such that either $x_{j}=x_{i}$ and $y_{j}=y_{i}-1$, or $x_{j}=x_{i}-1$ and $y_{j}=y_{i}$. This contradicts the choice of $i$.

Recall that $b_{0}$ and $b_{1}$ are two boundary tiles. 


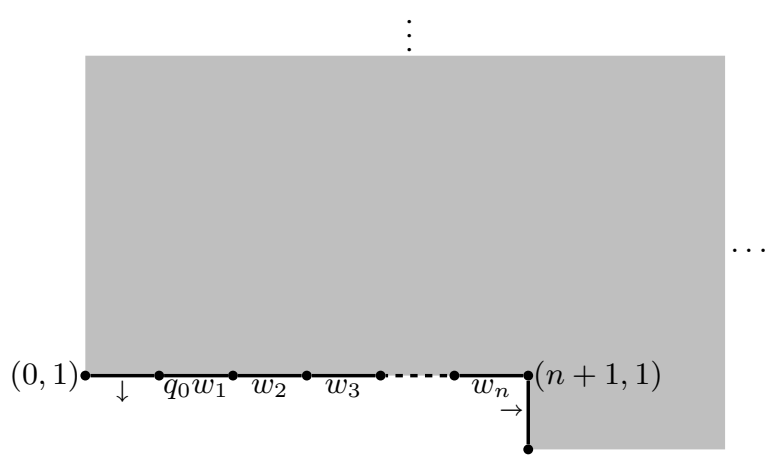

Fig. 2.

Claim 2. There exists $m \geq n+1$ such that the tiling sum $f$ in (2) can be written as

$$
f=\sum_{i=n+1}^{m-1} \tau_{i, 0} \llbracket b_{0} \rrbracket+\tau_{m, 0} \llbracket b_{1} \rrbracket+g_{1},
$$

where $g_{1}$ is a tiling sum, which does not contain a summand of the form $\tau_{x, 0} \llbracket t \rrbracket$ for some $x \in \mathbb{Z}$ and some tile $t$.

Since $f_{w}(\{(n+1,1),(n+1,0)\}, \rightarrow)=1, f$ must contain a summand of the form $\tau_{x, 0} \llbracket t \rrbracket$ with $x \geq n+1$. Let $m$ be the maximal $x$ with this property. The tile $t$ must be $b_{1}$, because every other tile has a color different from $c_{0}$ on its east side or on its south side. Then, $f$ would contain a summand of the form $\tau_{m+1,0} \llbracket t^{\prime} \rrbracket$ (which contradicts the choice of $m$ ) or $\tau_{m,-1} \llbracket t^{\prime} \rrbracket$ (which contradicts Claim 1). Hence, $f$ contains the summand $\tau_{m, 0} \llbracket b_{1} \rrbracket$. Now, by induction on $i$ we can easily show that $f$ must contain all summands $\tau_{i, 0} \llbracket b_{0} \rrbracket$ for $n+1 \leq i \leq m-1$. For this, note that $b_{0}$ is the only tile with color $\rightarrow$ on its east side. Hence, we can write $f$ as $f=\sum_{i=n+1}^{m-1} \tau_{i, 0} \llbracket b_{0} \rrbracket+\tau_{m, 0} \llbracket b_{1} \rrbracket+g_{1}$ for some tiling sum $g_{1}$. The diagram of the evaluation of the sum

$$
f_{1}=f_{w}+\sum_{i=n+1}^{m-1} \tau_{i, 0} \llbracket b_{0} \rrbracket+\tau_{m, 0} \llbracket b_{1} \rrbracket
$$

is shown in Figure 3. Note that we have

$$
f_{w}+f=f_{1}+g_{1}=0 .
$$

Now, assume that $g_{1}$ contains a summand of the form $\tau_{x, 0} \llbracket t \rrbracket$ for some $x$ and some tile $t$. Choose $x$ minimal with this property. Since the west or the south side of tile $t$ has a color different from $c_{0}$, the sum $g_{1}$ must contain a summand of the form $\tau_{x-1,0} \llbracket t^{\prime} \rrbracket$ (which contradicts the choice of $x$ ) or of the form $\tau_{x,-1} \llbracket t^{\prime} \rrbracket$ (which contradicts Claim 1). This proves Claim 2.

By Claims 1 and 2, we know that all summands in $g_{1}$ are of the form $\tau_{x, y} \llbracket t \rrbracket$ with $x \geq 0$ and $y \geq 1$. Moreover, $g_{1}$ added to $f_{1}$ in Figure 3 gives 0 . 


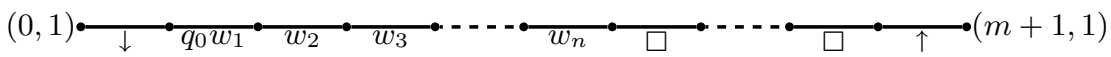

Fig. 3. The evaluation of the sum $f_{1}=f_{w}+\sum_{i=n+1}^{m-1} \tau_{i, 0} \llbracket b_{0} \rrbracket+\tau_{m, 0} \llbracket b_{1} \rrbracket$

Claim 3. The tiling sum $g_{1}$ does not contain a summand of the form $\tau_{x, y} \llbracket t \rrbracket(x \geq 0, y \geq$ 1) with $t \in\left\{b_{0}, b_{1}\right\}$.

Assume that $g_{1}$ contains the summand $\tau_{x, y} \llbracket t \rrbracket(x \geq 0, y \geq 1)$ with $t \in\left\{b_{0}, b_{1}\right\}$ and assume that $x$ is minimal with this property. Since the west edge of $t$ is labeled with $\rightarrow$, $g_{1}$ has to contain the summand $\tau_{x-1, y} \llbracket b_{0} \rrbracket$, which is again a contradiction.

Claim 4. The tiling sum $g_{1}$ does not contain a summand of the form $\tau_{x, y} \llbracket t \rrbracket$ with $x \geq$ $m+1$.

Assume that $g_{1}$ contains the summand $\tau_{x, y} \llbracket t \rrbracket$ with $x \geq m+1$ and assume that $y$ is minimal with this property. Since $t \notin\left\{b_{0}, b_{1}\right\}$ by Claim 3, the south side of $t$ is labeled with a color different from $c_{0}$. In order to match this up, $g_{1}$ has to contain also a summand of the from $\tau_{x, y-1} \llbracket t \rrbracket$, which contradicts the minimality of $y$.

Claim 5. For every position $(x, y)$ with $0 \leq x \leq m, y \geq 1$, the tiling sum $f$ does not contain a summand of the form $\tau_{x, y} \llbracket t \rrbracket+\tau_{x, y} \llbracket t^{\prime} \rrbracket$ (possibly with $t=t^{\prime}$ ), i.e., no position $(x, y)$ with $0 \leq x \leq m, y \geq 1$ receives two tiles.

Assume that $g_{1}=\tau_{x, y} \llbracket t \rrbracket+\tau_{x, y} \llbracket t^{\prime} \rrbracket+g_{1}^{\prime}(0 \leq x \leq m, y \geq 1)$, i.e., position $(x, y)$ receives at least two tiles. We can assume that $y$ is minimal with this property. Since $\left\{t, t^{\prime}\right\} \cap\left\{b_{0}, b_{1}\right\}=\emptyset$ by Claim 3, the south side of $t\left(t^{\prime}\right.$, resp.) is labeled with a color $c \neq c_{0}\left(c^{\prime} \neq c_{0}\right.$, resp. $)$. Hence, the edge $\{(x, y),(x+1, y)\}$ receives the colors $c$ and $c^{\prime}$ (we may have $c=c^{\prime}$, i.e., $\{(x, y),(x+1, y)\}$ receives the color $c$ twice). If $y \geq 2$, then we have to match this up by putting at least two tiles on position $(x, y-1)$. Since this contradicts the choice of $y$, we may assume that $y=1$. Recall that $0 \leq x \leq m$. Let $u_{0}$ and $u_{1}$ be two tiles that are put onto position $(x, 1)$ (we may have $u_{0}=u_{1}$ ). Since the south edges of $u_{0}$ and $u_{1}$ are labeled with colors different from $c_{0}$ and since the edge $\{(x, 1),(x+1,1)\}$ in Figure 3 is labeled with a single color exactly once, $g_{1}$ has to contain a summand of the form $\tau_{x, 0} f_{u}$ for some tile $u$. This contradicts Claim 2 and proves Claim 5.

Now that we have established Claims $1-5$, we are essentially faced with a classical tiling problem. We have to find a tiling (in the classical sense, where each grid point gets at most one tile), such that the south side of the final tiling is labeled with the line in Figure 3 and all other boundary edges are labeled with the distinguished color $c_{0}$. Note that the line in Figure 3 is labeled with the word $C_{1}=\downarrow q_{0} w_{1} w_{2} \cdots w_{n} \square^{m-n-1}$, which represents the initial configuration for the input $w=w_{1} w_{2} \cdots w_{n}$. Recall that we want to show that $M$ finally accepts the input $w$, which is equivalent to the fact that $M$ finally terminates on input $w$. In order to deduce a contradiction, assume that $M$ does not terminate on input $w$. Let $C_{i}(i \geq 1)$ be the unique configuration that is 


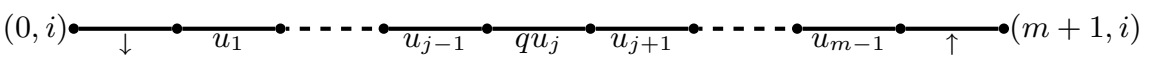

Fig. 4. The evaluation of the sum $f_{1}+h_{i}$

reached from $C_{1}$ after $i-1$ steps. We can view every $C_{i}$ as a word over the alphabet $\Gamma \cup(Q \times \Gamma) \cup\{\uparrow, \downarrow\}$ starting (ending) with $\downarrow(\uparrow)$. Here $\downarrow(\uparrow)$ marks the beginning (end) of the tape. The fact that the machine is in state $q$ with the tape head over the symbol $a$ is indicated by an occurrence of $(q, a)$ (which we abbreviate to $q a$ ) in $C_{i}$. By padding words with blanks, we can assume for every $i \geq 1$ : either $\left|C_{i}\right|=m+1$ (this is the case for $C_{1}$ ) or $\left|C_{i}\right|>m+1$ and $C_{i}$ does not end with $\square \uparrow$ (which means that $C_{i}$ cannot be represented by a shorter configuration word).

We prove by induction on $i$ that, for every $i \geq 1$, we have $\left|C_{i}\right|=m+1$ and the tiling sum $g_{1}$ in (3) can be written as $g_{1}=h_{i}+g_{i}$ where all summands in $h_{i}\left(g_{i}\right.$, resp.) are of the form $\tau_{x, y} \llbracket t \rrbracket$ with $0 \leq x \leq m$ and $1 \leq y \leq i-1(0 \leq x \leq m$ and $y \geq i$, resp.) and the diagram of the evaluation of $f_{1}+h_{i}$ is as shown in Figure 4, where $C_{i}=\downarrow u_{1} u_{2} \cdots u_{j-1} q u_{j} u_{j+1} \cdots u_{m-1} \uparrow$ with the $u_{i} \in \Gamma$. This will contradict the fact that $g_{1}$ is a finite tiling sum. It will therefore follow that $w$ is accepted by the machine.

For $i=1$ we take $h_{1}=0$. Assume that the above statement is already shown for $i \geq 1$. We have $0=f_{1}+g_{1}=\left(f_{1}+h_{i}\right)+g_{i}$, where the evaluation of the sum $f_{1}+h_{i}$ represents the configuration $C_{i}$ as shown in Figure 4. All summands in $g_{i}$ are of the form $\tau_{x, y} \llbracket t \rrbracket$ with $0 \leq x \leq m$ and $y \geq i$.

Note that it is not possible that $j=1$ and $\delta\left(q, u_{j}\right) \in Q \times \Gamma \times\{L\}$ (the machine $M$ is programmed in such way that it does not cross the left end of the tape). Moreover, $q$ is not the final state $q_{f}$ since we are assuming that $w$ is not accepted. We distinguish two cases. Suppose first $j=m-1$ and $\delta\left(q, u_{j}\right) \in Q \times \Gamma \times\{R\}$. Then the diagram of the evaluation of $f_{1}+h_{i}$ in fact has the following shape:

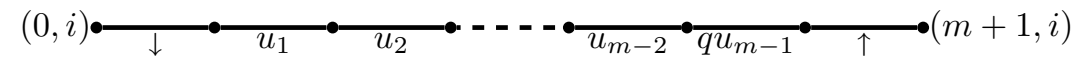

The only possible tiles that can be placed in position $(m-1, i)$ are action tiles with south side colored $q u_{m-1}$. Since the machine is deterministic and $\delta\left(q, u_{m-1}\right) \in Q \times \Gamma \times\{R\}$, the unique such action tile has the shape

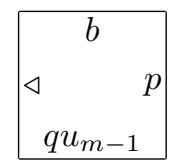

and so this tile must be placed in position $(m-1, i)$. But since there is no tile with west side $p \in Q$ and south side $\uparrow$, we obtain a contradiction thanks to Claim 5.

Next suppose that either $j<m-1$, or $j=m-1$ and $\delta\left(q, u_{j}\right) \in Q \times \Gamma \times\{L\}$. Then certainly $\left|C_{i+1}\right|=m+1$. Now, we can match up the edges in Figure 4 in exactly one way: In position $(j, i)$ we have to put the unique action tile with south side $q u_{j}$ (this tile is unique, since $M$ is deterministic). Depending on whether $\delta\left(q, u_{j}\right) \in Q \times \Gamma \times\{L\}$ 
or $\delta\left(q, u_{j}\right) \in Q \times \Gamma \times\{R\}$, we have to put one of the two merging tiles either to the left or to the right of the action tile. The rest of the row is filled up with alphabet tiles and the boundary tile $b_{7}\left(b_{2}\right.$, resp.) at position $(0, i)((m, i)$, resp.) (using that only these types of tiles have $\triangleleft$ on their east side or $\triangleright$ on their west side). The claims ensure no further tiles may be placed. In case $\delta\left(q, u_{j}\right)=(p, b, L)$, the tiling looks as in Figure 5 . We define $h_{i+1}$ as the sum of $h_{i}$ and all summands $\tau_{x, i} \llbracket t(x, i) \rrbracket$, where $0 \leq x \leq m$

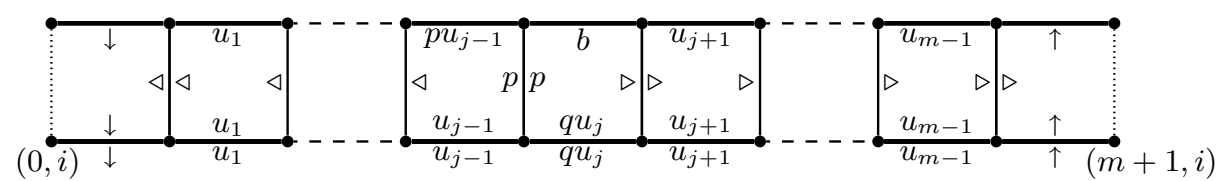

Fig. 5. Simulating a move of the Turing machine

and $t(x, i)$ is the unique tile that we put on position $(x, i)$. The tiling sum $g_{i+1}$ is $g_{i}$ without these summands $\tau_{x, i} \llbracket t(x, i) \rrbracket$. We now have shown that $w$ is accepted by $M$ if there exists a tiling sum $f$ with $f_{w}+f=0$.

For the other direction, we assume that $w$ is accepted by $M$. We have to show that there exists a tiling sum $f$ with $f_{w}+f=0$. This is much easier than what we have already done. Since $w$ is accepted by $M$, there exists a number $m-1$ (the space consumption of $M$ on input $w$ ) and sequence of configurations (encoded as before) $C_{1}, C_{2}, \ldots, C_{N}$ (all of length $m+1$ ) such that $C_{1}=\downarrow q_{0} w \square^{m-n-1} \uparrow$ is the initial configuration for the input $w, C_{N}$ is of the form $\downarrow q_{f} \square^{m-1} \uparrow$, and $M$ moves from $C_{i}$ to $C_{i+1}$ in one step $(1 \leq i \leq N-1)$. From this computation we can build up a tiling in the standard way (every position receives at most one tile) to obtain the tiling sum $f$, essentially by reversing the previous argument. Namely, we first add to $f_{w}$ the sum $\sum_{i=n+1}^{m-1} \tau_{i, 0} \llbracket b_{0} \rrbracket+\tau_{m, 0} \llbracket b_{1} \rrbracket$ to obtain Figure 3 . Then one continues as per Figure 5 to build up rows 2 through $N$. In this way one obtains a sum $f_{w}+g$, with $g$ a tiling sum, whose evaluation looks like:

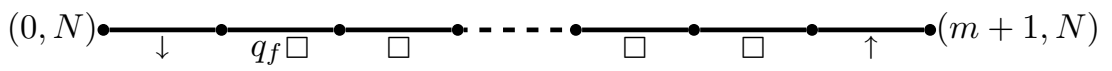

Finally, we complete the tiling as follows.

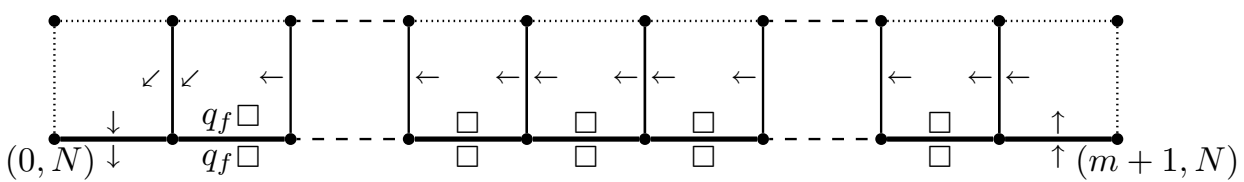

Formally, $f=g+\tau_{(0, N)} \llbracket b_{6} \rrbracket+\tau_{(1, N)} \llbracket b_{5} \rrbracket+\sum_{i=2}^{m-1} \tau_{(i, N)} \llbracket b_{4} \rrbracket+\tau_{(m, N)} \llbracket b_{3} \rrbracket$ is a tiling sum with $f_{w}+f=0$. This completes the proof of Theorem 7 . 
We now proceed to the proof of Theorem 4, thereby establishing Corollary 5 and Theorem 6. We recall that if $G$ is a group, then the free $G$-module on a set $X$ can be realized as the abelian group of all finitely supported functions $f: G \times X \rightarrow \mathbb{Z}$ with pointwise addition and module action given by $g_{0} f(g, x)=f\left(g_{0}^{-1} g, x\right)$.

Proof (Theorem 4). The abelian group $M$ of all finitely supported functions from $\mathscr{E} \times C$ to $\mathbb{Z}$ is a free $\mathbb{Z} \times \mathbb{Z}$ module of rank $2|C|$ via the translation action. Indeed, let us set $r=\{(0,0),(1,0)\}$ and $u=\{(0,0),(0,1)\}$. Then $\mathscr{E} \times C=(\mathbb{Z} \times \mathbb{Z}) \times\{r, u\} \times C$ since each horizontal edge is uniquely of the form $(a, b)+r$ and each vertical edge is uniquely of the form $(a, b)+u$. A tiling sum is precisely an element of the subsemimodule $N$ of $M$ generated by the set $\{\llbracket t \rrbracket \mid t \in T\}$. Then the zero tiling sum problem is asking exactly whether there exists $f \in N$ so that $f_{0}+f=0$, which is equivalent to asking whether $-f_{0} \in N$. Theorem 7 provides a fixed tiling system with undecidable zero tiling sum problem. Therefore this is a fixed finitely generated subsemimodule of a fixed free $(\mathbb{Z} \times \mathbb{Z})$-module with undecidable subsemimodule membership problem. This completes the proof.

\section{Rational subsets of two-dimensional lamplighter groups}

By a two-dimensional lamplighter group, we mean a wreath product of the form $\mathbb{Z} / n \mathbb{Z}$ ? $(\mathbb{Z} \times \mathbb{Z})$ for $n \geq 2$. In this section, we show that the rational subset membership problem is undecidable for such groups. By an effective ring, we mean a unital ring $R$ whose arithmetic can be represented effectively (like $\mathbb{Z}$ or $\mathbb{Z} / n \mathbb{Z}$ ). Let $G$ be a finitely generated group with generating set $\Sigma$. The subset sum problem for a finitely generated $R G$ module $M$ is the following algorithmic problem. Given $m \in M$ and a finite subset $F \subseteq$ $M$ of non-zero elements, determine whether there exist distinct elements $g_{1}, \ldots, g_{n} \in$ $G$ and elements $f_{1}, \ldots, f_{n} \in F$ (not necessarily distinct) so that $m=\sum_{i=1}^{n} g_{i} f_{i}$. In the case the answer is "yes", we say that $m$ is a subset sum of $F$. If $F$ is fixed, then we call this the subset sum problem for $F$.

Theorem 8. Let $R \neq 0$ be an effective ring. Then there is a free $R(\mathbb{Z} \times \mathbb{Z})$-module of finite rank and a fixed finite subset $F$ of non-zero elements so that the subset sum problem for $F$ is undecidable.

Proof. Let $T$ be the fixed tiling system with undecidable zero tiling sum problem constructed earlier. We now consider mappings $f: \mathscr{E} \times C \rightarrow R$ instead of mappings to $\mathbb{Z}$, but otherwise retain the definitions and notation from the proof of Theorem 7 . The proof of that theorem shows that $w$ is accepted by the Turing machine if and only if $-f_{w}$ is a subset sum of $F=\{\llbracket t \rrbracket \mid t \in T\}$. Indeed, the proof shows that if $w$ is accepted by the Turing machine, then there is a tiling sum $f$ in which no two tiles are placed in the same position and so that $f_{w}+f=0$. Conversely, if $-f_{w}$ is a subset sum of $F$, then we can write $f_{w}+f=0$ with $f$ a tiling sum never placing two tiles in the same position. The argument of Theorem 7 now shows that $w$ must be accepted by the Turing machine, the only difference being that Claim 5 is now an assumption rather than a result that must be proved. 
We now aim to show that two-dimensional lamplighter groups have undecidable rational subset membership problem.

Proposition 9. Let $R=\mathbb{Z} / n \mathbb{Z}(n \geq 2)$ and suppose that $M$ is a finite rank free $R[\mathbb{Z} \times$ $\mathbb{Z}]$-module with fixed finite subset $F$ of non-zero elements having an undecidable subset sum problem. Then there is a fixed rational subset of $M \rtimes(\mathbb{Z} \times \mathbb{Z})$ with undecidable membership problem.

Proof. Let $B$ be a basis for $M$ and take as a generating set for $G=M \rtimes(\mathbb{Z} \times \mathbb{Z})$ the set $B \cup\{x, y\}$ where $x=(1,0)$ and $y=(0,1)$. We claim that $m \in M$ is a subset sum of $F$ if and only if $(m,(0,0))$ belongs to the rational subset

$$
L=\left\{x^{ \pm 1}, y^{ \pm 1}\right\}^{*}\left[(x \cup F x)^{*} y\left(x^{-1}\right)^{*}\right]^{*}\left\{x^{ \pm 1}, y^{ \pm 1}\right\}^{*} .
$$

Let us give a high level description of how this works. The first term $\left\{x^{ \pm 1}, y^{ \pm 1}\right\}^{*}$ in $L$ lets us move to any position in $\mathbb{Z} \times \mathbb{Z}$. Then $(x \cup F x)^{*}$ lets us move to the right or add an element of $F$ translated to the current position and then move right. The term $y\left(x^{-1}\right)^{*}$ allows us to move up one row and then move as far left as needed. Now we keep repeating until we are done translating elements of $F$ in positions. Then we use $\left\{x^{ \pm 1}, y^{ \pm 1}\right\}^{*}$ to return to the origin. Notice that when following this procedure, a position can have at most one element of $F$ translated to it.

For instance, suppose $m=x^{i_{1}} y^{j_{1}} f_{i_{1}, j_{1}}+\cdots+x^{i_{k}} y^{j_{k}} f_{i_{k}, j_{k}}$ is a subset sum of $F$ where $\left(i_{1}, j_{1}\right)<\left(i_{2}, j_{2}\right)<\cdots<\left(i_{k}, j_{k}\right)$ in right lexicographical order (i.e., $(a, b)<$ $(c, d)$ if $b<d$, or $b=d$ and $a<c)$. Then we begin with the product $x^{i_{1}} y^{j_{1}}$ from $\left\{x^{ \pm 1}, y^{ \pm 1}\right\}^{*}$ to get to the starting point of our sum. Then using $\left[(x \cup F x)^{*} y\left(x^{-1}\right)^{*}\right]^{*}$ we build up row by row, always going upward, an element of the form $(m,(a, b))$. Finally we multiply by $x^{-a} y^{-b} \in\left\{x^{ \pm 1}, y^{ \pm 1}\right\}^{*}$ to obtain $(m, 0)$. Conversely, any element of the form $(m, 0)$ belonging to $L$ must have $m$ a subset sum of $F$ since the regular expression $L$ never permits you to translate by the same element of $\mathbb{Z} \times \mathbb{Z}$ twice.

Now we can argue as before to obtain undecidability for the two-dimensional lamplighther groups.

Theorem 10. Rational subset membership is undecidable for a fixed rational subset of $\mathbb{Z} / n \mathbb{Z} \imath(\mathbb{Z} \times \mathbb{Z})$ for any $n \geq 2$.

Proof. Again write $R=\mathbb{Z} / n \mathbb{Z}$. Then $\mathbb{Z} / n \mathbb{Z} \imath(\mathbb{Z} \times \mathbb{Z})=R[\mathbb{Z} \times \mathbb{Z}] \rtimes(\mathbb{Z} \times \mathbb{Z})$. By Theorem 8 , there is a free $R[\mathbb{Z} \times \mathbb{Z}]$-module $M$ of rank $m$ with an undecidable subset sum problem for a fixed finite subset $F$. Since $R[\mathbb{Z} \times \mathbb{Z}]$ is a free $R[m \mathbb{Z} \times \mathbb{Z}]$-module of rank $m$, we can embed $M \rtimes(\mathbb{Z} \times \mathbb{Z})$ in $\mathbb{Z} / n \mathbb{Z} \imath(\mathbb{Z} \times \mathbb{Z})$. The result now follows from Proposition 9.

As a corollary, it follows that $G \imath(\mathbb{Z} \times \mathbb{Z})$ has an undecidable rational subset membership problem for any non-trivial group $G$.

Corollary 11. Let $G$ be a non-trivial group. Then $G ?(\mathbb{Z} \times \mathbb{Z})$ has an undecidable rational subset membership problem for a fixed rational subset.

Proof. Either $G \imath(\mathbb{Z} \times \mathbb{Z})$ contains a copy of $\mathbb{Z} \imath(\mathbb{Z} \times \mathbb{Z})$ or of $\mathbb{Z} / n \mathbb{Z} \imath(\mathbb{Z} \times \mathbb{Z})$. 
The argument of Theorem 10 can be adapted to show that membership is undecidable for a fixed rational subset of the free group of rank 2 in the variety $\mathfrak{A}(n) \cdot \mathfrak{A}$, where $\mathfrak{A}(n)$ (resp. $\mathfrak{A}$ ) is the variety of abelian groups of exponent $n$ (resp. of all abelian groups). The adaptations are entirely analogous to those used in going from submonoid membership for $\mathbb{Z} \imath(\mathbb{Z} \times \mathbb{Z})$ to submonoid membership for the free metabelian group of rank 2 .

\section{References}

1. J. Almeida. Semidirect products of pseudovarieties from the universal algebraist's point of view. Journal of Pure and Applied Algebra, 60(2):113-128, 1989.

2. A. W. Anissimov and F. D. Seifert. Zur algebraischen Charakteristik der durch kontext-freie Sprachen definierten Gruppen. Elektron. Informationsverarbeit. Kybernetik, 11(10-12):695702, 1975.

3. J. Avenhaus and D. Wißmann. Using rewriting techniques to solve the generalized word problem in polycyclic groups. In Proceedings of the ACM-SIGSAM 1989 International Symposium on Symbolic and Algebraic Computation, pages 322-337. ACM Press, 1989.

4. G. Baumslag, F. B. Cannonito, and D. J. S. Robinson. The algorithmic theory of finitely generated metabelian groups. Transactions of the American Mathematical Society, 344(2):629648, 1994.

5. M. Benois. Parties rationnelles du groupe libre. C. R. Acad. Sci. Paris, Sér. A, 269:11881190, 1969.

6. R. Berger. The undecidability of the domino problem. Mem. Amer. Math. Soc. No., 66:72, 1966.

7. J. Berstel. Transductions and context-free languages. Teubner Studienbücher, Stuttgart, 1979.

8. E. Börger, E. Grädel, and Y. Gurevich. The classical decision problem. Universitext. Springer-Verlag, Berlin, 2001.

9. K. Brown. Cohomology of Groups. Springer, 1994.

10. S. Eilenberg and M. P. Schützenberger. Rational sets in commutative monoids. Journal of Algebra, 13:173-191, 1969.

11. R. H. Gilman. Formal languages and infinite groups. In Geometric and computational perspectives on infinite groups (Minneapolis, MN and New Brunswick, NJ, 1994), volume 25 of DIMACS Ser. Discrete Math. Theoret. Comput. Sci., pages 27-51. Amer. Math. Soc., Providence, RI, 1996.

12. M. Kambites, P. V. Silva, and B. Steinberg. On the rational subset problem for groups. Journal of Algebra, 309(2):622-639, 2007.

13. M. Lohrey and B. Steinberg. The submonoid and rational subset membership problems for graph groups. Journal of Algebra, 320(2):728-755, 2008.

14. A. I. Malcev. On homomorphisms onto finite groups. American Mathematical Society Translations, Series 2, 119:67-79, 1983. Translation from Ivanov. Gos. Ped. Inst. Ucen. Zap. 18 (1958) 49-60.

15. S. W. Margolis, J. Meakin, and Z. Šuniḱ. Distortion functions and the membership problem for submonoids of groups and monoids. In Geometric methods in group theory, volume 372 of Contemp. Math., pages 109-129. Amer. Math. Soc., Providence, RI, 2005.

16. A. Myasnikov, V. Roman'kov, A. Ushakov, and A.Vershik. The word and geodesic problems in free solvable groups. Technical report, arXiv.org, 2008. http: //arxiv.org/abs/0807.1032. 
17. M. Nedbaj. The occurrence problem in a rational subset of the free product of groups. Vestn. Omsk. Univ., 2000(2):17-18, 2000.

18. R. M. Robinson. Undecidability and nonperiodicity for tilings of the plane. Inventiones Mathematicae, 12:177-209, 1971.

19. V. Roman'kov. On the occurence problem for rational subsets of a group. In V. Roman'kov, editor, International Conference on Combinatorial and Computational Methods in Mathematics, pages 76-81, 1999.

20. V. A. Roman'kov. Equations in free metabelian groups. Akademiya Nauk SSSR. Sibirskoe Otdelenie. Sibirskiŭ Matematicheskiu Zhurnal, 20(3):671-673, 694, 1979.

21. N. S. Romanovski1. Some algorithmic problems for solvable groups. Algebra i Logika, 13(1):26-34, 1974.

22. N. S. Romanovskiur. The occurrence problem for extensions of abelian groups by nilpotent groups. Sibirsk. Mat. Zh., 21:170-174, 1980.

23. C. Sims. Computation with finitely presented groups. Cambridge University Press, 1994.

24. U. U. Umirbaev. The occurrence problem for free solvable groups. Sibirskĭ Fond Algebry $i$ Logiki. Algebra i Logika, 34(2):211-232, 243, 1995. 